Revue internationale de l'économie sociale

Recma

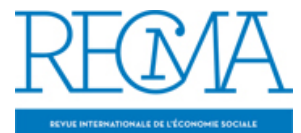

\title{
La dimension politique de l'économie sociale
}

\section{Jean-François Draperi}

Numéro 341, juillet 2016

URI : https://id.erudit.org/iderudit/1037430ar

DOI : https://doi.org/10.7202/1037430ar

Aller au sommaire du numéro

Éditeur(s)

Association Recma

ISSN

1626-1682 (imprimé)

2261-2599 (numérique)

Découvrir la revue

Citer ce document

Draperi, J.-F. (2016). La dimension politique de l'économie sociale. Revue internationale de l'économie sociale, (341), 4-5.

https://doi.org/10.7202/1037430ar d'utilisation que vous pouvez consulter en ligne.

https://apropos.erudit.org/fr/usagers/politique-dutilisation/ 


\title{
LA DIMENSION POLITIQUE DE L'ÉCONOMIE SOCIALE
}

\begin{abstract}
A u moment où disparaît l'homme politique français à qui l'économie sociale doit d'être aujourd'hui reconnue, celle-ci est amenée à prendre l'une de ses premières positions politiques importantes. La loi travail questionne en effet les rapports entre la liberté du travail et l'organisation collective. Son article 2 affirme: "L L primauté de l'accord d'entreprise en matière de durée du travail devient le principe de droit commun ». L'accord d'entreprise prime dorénavant sur l'accord de branche et inverse la hiérarchie des normes en vigueur.
\end{abstract}

\section{Un écho de la loi Le Chapelier}

Il est difficile de ne pas rapprocher cet article du préambule à la loi Le Chapelier du 14 juin 1791, qui affirme: "Il faut donc remonter au principe que c'est aux conventions libres, d'individus à individus, à fixer la journée pour chaque ouvrier; c'est ensuite à l'ouvrier à maintenir la convention qu'il a faite avec celui qui l'occupe."

En 1791, les décrets d'Allarde et de Le Chapelier ont pour objet de mettre fin aux révoltes ouvrières. Une émeute sévèrement réprimée en avril 1789 (affaire Réveillon), qui fit plusieurs centaines de morts au faubourg Saint-Antoine à Paris - soit bien plus que la prise de la Bastille -, avait marqué les esprits. Le 15 février, le baron d'Allarde, se saisissant des arguments de l'Edit de Turgot (24 février 1776), fit prendre un décret supprimant les maîtrises et jurandes. Face à l'agitation ouvrière persistante, le maire de Paris, Jean-Sylvain Bailly, adressa un "Avis aux ouvriers » le 26 avril 1791: "Le corps municipal est instruit que des ouvriers de quelques professions se réunissent journellement en très grand nombre, se coalisent au lieu d'employer leur temps au travail, délibèrent et font des arrêtés par lesquels ils taxent arbitrairement le prix de leurs journées » (cité par Jean Bennet, La Mutualitéfrançaise, des origines à la Révolution de 1789, Ciem, 1981, p. 739). Prenant peur, l’Assemblée Nationale confie à Guy le Chapelier, député de Rennes et avocat, de rédiger un projet de loi dont les deux premiers articles énoncent « L'anéantissement de toutes espèces de corporations de citoyens du même état et profession " (Article 1). "Les citoyens d'un même état ou profession, les entrepreneurs, ceux qui ont boutique ouverte, les ouvriers d'un art quelconque, ne pourront, lorsqu'ils se trouveront ensemble, se nommer ni président, ni secrétaires, ni syndic, tenir des registres, prendre des arrêtés ou délibérations, former des règlements sur leurs prétendus intérêts communs» (Article 2).

Michel Vovelle commente: "Au conflit de deux libertés, la libertéd'association contre la liberté du travail, l'arbitrage de la Constituante fait prévaloir celle qui est la plus favorable à la bourgeoisie» (Michel Vovelle, La chute de la monarchie 1787-1792, Le Seuil, 1972, p. 174). 
La loi Le Chapelier s'inscrit en effet dans une série d'interdits: elle vise les compagnonnages, c'est-à-dire les associations de citoyens exerçant la même profession, alors que le décret d'Allarde pris le 15 février 1791 visait les corporations. L’Edit de Necker du 23 août 1776 avait interdit les confréries, dont certaines s'étaient transformées en sociétés de secours mutuels dans les dernières décennies du XVIII ${ }^{e}$ siècle. Celles qui, parmi ces sociétés, gardaient un caractère religieux furent interdites par le décret du 18 août 1792 relatif à la suppression des congrégations séculières et des confréries.

\section{Liberté du travail et liberté d'association}

Considérant que la liberté du travail ne va pas sans la liberté d'association, l'économie sociale et solidaire (ESS) se situe au croisement de ces deux libertés. Si la responsabilité individuelle est revendiquée, elle affirme également que l'émancipation ne peut venir du seul rapport interindividuel, que celui-ci soit un rapport de force ou un rapport de solidarité. C’est sans doute essentiellement en ce sens que l'ESS est irréductible à la pensée libérale: loin de se suffire du marché et de l'Etat, la régulation sociale ne peut se priver de l'association, ce que l'on théorise désormais volontiers par le concept de « commun ».

C'est en substance ce qu'exprime l'Union des syndicats de l'économie sociale (Udes) sur la loi travail en affirmant: "La branche doit conserver son rôle de régulation au sein d'un secteur d'activité. C'est au niveau de la branche que doivent se décider les mesures relatives au temps de travail: temps partiel, majoration des heures supplémentaires, travail de nuit, durée maximale de travail quotidienne et hebdomadaire [...]. Ceciévitera un nivellement par le bas et l'accroissement des disparités dans les territoires. Car, si les entreprises ont la possibilité de mettre en place des dispositions moins favorables que celles de l'accord de branche, l'UDES redoute l'émergence de distorsions importantes dans certains secteurs sous contraintes financières fortes, où les fonds publics se raréfient. C'est notamment le cas dans les secteurs de l'aide et des soins à domicile et du social et médico-social. En revanche, l'accord d'entreprise peut primer sur l'accord de branche lorsqu'il concerne des sujets impactant l'organisation quotidienne et spécifique d'une entreprise, comme les congés et les astreintes " (Hugues Vidor, président, site de l'Udes).

Jusqu'à cette date et sauf exception, les instances représentatives de l'économie sociale se sont peu exprimées lors des grands débats nationaux ou internationaux. Même s'il est vrai qu'une position et une seule risque de ne pas refléter la diversité des composantes représentées, nul ne niera la dimension politique de l'économie sociale: comment un mouvement revendiquant des valeurs fondatrices pourrait-il éviter de se situer face à des choix de société qu'expriment les responsables politiques? Michel Rocard le savait bien, qui écrivait: "C'est bien de l'économie sociale qu'émerge tout à la fois un art de produire respectueux de la démocratie et de la dignité de ceux qui y contribuent et une sélection d'activités et de produits construisant un monde plus généreux, plus solidaire et plus sûr de sa pérennitéécologique " $\left(\right.$ Recma $\left.\mathrm{n}^{\circ} 282,2001\right)$. Nous reviendrons dans un proche avenir sur la pensée et l'action de celui qui eut, le premier de notre époque, une vision politique de l'économie sociale.

JEAN-FRANÇOIS DRAPERI 